\title{
COMPARAISON DU TRAITEMENT LEXICOGRAPHIQUE DES APPELLATIONS DES IDENTITÉS DE GENRE NON TRADITIONNELLES DANS LES DICTIONNAIRES PROFESSIONNELS ET PROFANES
}

\section{Mireille Elchacar}

Klincksieck | «Éla. Études de linguistique appliquée »

2019/2 N 194 | pages 177 à 191

ISSN 0071-190X

ISBN 9782252042731

Article disponible en ligne à l'adresse :

https://www.cairn.inforevue-ela-2019-2-page-177.htm

Distribution électronique Cairn.info pour Klincksieck.

(C) Klincksieck. Tous droits réservés pour tous pays.

La reproduction ou représentation de cet article, notamment par photocopie, n'est autorisée que dans les limites des conditions générales d'utilisation du site ou, le cas échéant, des conditions générales de la licence souscrite par votre établissement. Toute autre reproduction ou représentation, en tout ou partie, sous quelque forme et de quelque manière que ce soit, est interdite sauf accord préalable et écrit de l'éditeur, en dehors des cas prévus par la législation en vigueur en France. Il est précisé que son stockage dans une base de données est également interdit. 


\section{COMPARAISON DU TRAITEMENT LEXICOGRAPHIQUE DES APPELLATIONS DES IDENTITÉS DE GENRE NON TRADITIONNELLES DANS LES DICTIONNAIRES PROFESSIONNELS ET PROFANES ${ }^{1}$}

Résumé : Depuis le début des années 2000, et avec une présence plus forte ces dernières années, on voit apparaître des néologismes pour nommer les appellations d'identité de genre et d'orientation sexuelle non traditionnelles (LGBT, queer, trans, etc.). Certaines de ces appellations ont pénétré la nomenclature de dictionnaires professionnels. En parallèle, des lexiques profanes de ce vocabulaire ont vu le jour. Ce texte propose une analyse de la nomenclature et du traitement définitoire de ce vocabulaire dans des lexiques profanes et dans quelques dictionnaires professionnels.

Depuis quelques années, des appellations ont été créées pour désigner des identités de genre et des orientations sexuelles non traditionnelles. Des appellations établies depuis plus longtemps telles gay, lesbienne, homosexuel, etc. côtoient aujourd'hui LGBT, trans, queer et autres néologismes. La création de ces appellations témoigne d'une volonté d' " appropriation identitaire de dénominations par des groupes sociaux, à des fins de reconnaissance » (Petit, 2012 : 5). Nous avons procédé précédemment à une analyse lexicologique des diverses appellations en circulation dans les médias grand public (Elchacar et Salita, 2018). Pour la présente étude, nous nous intéressons à leur description lexicographique.

Parmi les appellations proposées par les groupes militants, certaines resteront confinées à ces milieux, d'autres perceront dans la presse générale, et d'autres enfin accèderont à la nomenclature des dictionnaires généraux, malgré leur caractère récent. La présence de certaines nouvelles appellations à la nomenclature de dictionnaires professionnels n'a pas empêché plusieurs glossaires et lexiques profanes de voir le jour. Pourquoi? Si les groupes domi-

1. Nous tenons à remercier Caroline Dubois pour sa lecture attentive de cet article. 
nés ${ }^{2}$ ressentent le besoin de rédiger leurs propres glossaires, est-ce parce qu'ils ne se sentent pas adéquatement représentés dans les ouvrages de référence officiels ${ }^{3}$ ? Nous proposons une analyse de la nomenclature et du traitement définitoire dans les deux types de dictionnaires.

\section{QUELQUES PRÉCISIONS}

Nous reprenons la distinction de Vincent entre dictionnaires professionnels et profanes :

Les dictionnaires professionnels sont donc produits par des lexicographes œuvrant au sein d'institutions publiques ou privées alors que les dictionnaires profanes émanent d'entreprises privées et sont rédigés par des non-lexicographes qui peuvent venir d'horizons variés, incluant la linguistique, mais sans spécialité en lexicographie. (Vincent, 2017)

Une autre distinction importante se situe à l'intérieur du champ lexical à l'étude. On identifie deux catégories principales : les appellations des identités de genre, aussi appelées «expression de genre » (ex. : trans), et celles des orientations sexuelles (ex. : homosexuel). La frontière est poreuse, certaines appellations pouvant se retrouver dans les deux catégories, comme $L G B T$ ou queer. La plupart des appellations dont il sera question ici appartiennent à la catégorie des identités de genre non traditionnelles. Dans ce texte, et pour éviter des lourdeurs, nous utiliserons donc « appellations des identités de genre non traditionnelles » lorsque nous ferons référence aux néologismes à l'étude.

\section{LES DICTIONNAIRES ET LEXIQUES RETENUS}

Les ouvrages retenus sont de natures diverses et répondent à des visées différentes. Notre objectif n'est pas de comparer chaque élément entre les divers dictionnaires à l'étude, mais plutôt de voir si la nomenclature établie par chacun est représentative de la circulation des appellations dans la presse générale, et si le traitement définitoire est satisfaisant pour cerner le référent.

Du côté des dictionnaires professionnels, nous avons retenu trois ouvrages consultables en ligne. Pour le Québec, nous avons choisi le dictionnaire Usito ${ }^{4}$, dictionnaire général du français québécois accessible sur abonnement (individuel ou institutionnel, d'un établissement scolaire ou universitaire par exemple), et Le grand dictionnaire terminologique ${ }^{5}$, produit par l'Office québécois de la langue française (OQLF), accessible gratuitement à toute la population. Du

2. Nous utilisons ici des éléments du cadre théorique de Pierre Bourdieu, que nous n'étayerons pas, faute d'espace.

3. Se pose également la question de savoir si l'usager a le réflexe de consulter les dictionnaires professionnels lors de ses recherches, en ligne ou non. Il nous est impossible de répondre ici à cette question.

4. www.usito.com

5. www.granddictionnaire.com 
côté des ouvrages hexagonaux, nous avons retenu le Nouveau Petit Robert électronique, accessible sur abonnement.

Puisqu'ils sont sans doute moins connus, nous présenterons les lexiques profanes plus en détail. Les quatre lexiques retenus sont les suivants : Le vocabulaire LGBTIQ en français du Wiktionnaire, le Lexique LGBT sur la diversité sexuelle et de genre en milieu de travail de la Chambre de commerce LGBT du Québec, Le glossaire de la Fédération nationale des enseignantes et enseignants du Québec intitulé LGBTQI2SNBA+. Les mots de la diversité liée au sexe, au genre et à l'orientation sexuelle, et le Glossaire des termes $L G B T$ du Rainbow Project, projet éducatif de la Communauté européenne.

Le Wiktionnaire consacre un lexique à part au « vocabulaire LGBTIQ en français $»^{6}$ (nous y référerons en tant que Wiktionnaire dans le présent texte). Il s'agit donc d'entrées indépendantes de celles auxquelles on peut avoir accès directement à partir du Wiktionnaire. Ce lexique est le plus complet de notre corpus, pour la nomenclature, avec 133 entrées, mais également en ce qui concerne le traitement lexicographique, avec de nombreuses rubriques, comme c'est la norme dans le Wiktionnaire, et comme on peut l'observer avec n'importe quel article qu'on consulterait au hasard. Cependant, au niveau des textes de présentation, le Wiktionnaire fournit peu d'informations. Il explique simplement ceci quant au choix des entrées ${ }^{7}$ :

Cette catégorie regroupe les termes relatifs, propres ou apparentés aux thèmes ou communautés lesbienne, gay, bisexuelle, transgenre, intersexe ou queer (allosexuelle/ altersexuelle). Certains mots font partie du langage courant, d'autres relèvent plus de l'argot de ces populations.

Aucune information n'est fournie concernant le lieu de production; on trouve à la nomenclature autant des appellations propres au Québec (bispirituel, allosexuel) que propres à la France ou à l'Europe (transpédégouine).

Le lexique de la Chambre de commerce LGBT du Québec ${ }^{8}$ (CCGQ) a été rédigé avec l'objectif de fournir des outils de rédaction au monde du travail :

Nous avons entrepris ce projet de lexique en 2013 afin de répondre à un besoin grandissant en milieu de travail, soit l'adoption d'un vocabulaire juste et inclusif pour exprimer les réalités LGBT dans les normes d'emploi, les conditions de travail, les conventions collectives, les avantages sociaux et les rapports entre employés, cadres, syndicats et associations professionnelles.

La réflexion ayant mené au projet est expliquée en lien avec les néologismes mais également l'anglais : « les questions de la diversité en milieu de travail prennent de plus en plus d'importance au sein des entreprises et de nombreux termes sont mal adaptés en français, mal traduits ou sont carrément sans équivalence. »

6. https://fr.wiktionary.org/wiki/Cat\%C3\%A9gorie:Vocabulaire_LGBTIQ_en_fran\%C3\%A7ais

7. Tous les extraits des lexiques sont tirés de leurs sites Web. Nous ne répéterons pas l'adresse en guise de référence à chaque citation.

8. https://cclgbtq.org/wp-content/uploads/2015/12/Lexique-LGBT.pdf. À noter qu'au moment de la publication du guide, le regroupement s'appelait la Chambre de commerce gaie du Québec. 
Le Glossaire de la Fédération nationale des enseignantes et enseignants du Québec ${ }^{9}$ (FNEEQ) répond à une visée éducative, clairement perceptible à travers les textes de présentation de l'ouvrage : « Nous ne cherchons pas à statuer définitivement sur la terminologie, mais plutôt à dresser un portrait des différentes réalités qui soit utile au rôle d'enseignantes et d'enseignants. » Les entrées se présentent sous la forme de petits paragraphes, en texte suivi plutôt qu'en employant le métalangage lexicographique habituel : «Une personne pansexuelle est attirée sexuellement par les personnes indépendamment de leur genre. »

Enfin, le glossaire produit dans le cadre du Rainbow Project ${ }^{10} \mathrm{~s}^{\prime}$ inscrit lui aussi dans une perspective éducative : «L'objectif est [...] d'enrayer l'homophobie/transphobie en étudiant les stéréotypes et en les confrontant à des outils éducatifs ». La manière de présenter les entrées est à mi-chemin entre le traitement définitoire classique et le texte suivi : «Lesbienne (n. et adj.) Terme utilisé pour désigner une femme émotionnellement et physiquement attirée par une autre femme. »

\section{LES ANALYSES}

\section{1. La nomenclature}

Puisqu'elle est spécialisée, on pourrait s'attendre à ce que la nomenclature des dictionnaires profanes soit plus étendue que celle des dictionnaires professionnels, généraux. Mais les nomenclatures ne sont pas forcément mieux construites parce qu'elles sont établies par des personnes issues des groupes concernés, qui n'appliqueront pas nécessairement tous les principes lexicographiques.

Pour juger de la représentativité de la nomenclature des ouvrages à l'étude, nous avons d'abord vérifié que les appellations d'identité de genre non traditionnelle les plus fréquentes au Québec étaient présentes. Nous nous sommes appuyée sur les fréquences de ces appellations dans la presse générale au Québec : nous avons retenu les appellations d'identité de genre ou d'orientation sexuelle non traditionnelle qui ont été repérées dans au moins 100 documents dans les sources canadiennes francophones de la banque de données Eureka (appelée Infopresse en Europe). Pour lancer nos recherches, nous avons pris comme point de départ les nomenclatures des lexiques profanes eux-mêmes, et les avons comparées entre elles, ainsi que les données de notre précédente étude sur les appellations d'identité de genre non traditionnelle dans la presse générale québécoise (Elchacar et Salita, 2018). Comme notre intérêt porte sur la volonté des groupes dominés de se voir désignés par des appellations correspondant à leur identité de genre, nous avons laissé de côté tout le vocabulaire injurieux ou les mots qui ne sont pas une appellation de personnes ou de groupes (comme homoparentalité).

9. http://fneeq.qc.ca/wp-content/uploads/Glossaire.pdf

10. http://www.rainbowproject.eu/material/fr/glossary.htm 


\begin{tabular}{|l|c|c|c|c|c|c|c|}
\hline & PRE & Usito & GDT & Wiktionnaire & FNEEC & CCGQ & Rainbow \\
\hline Allosexuel & 0 & 1 & 1 & 1 & 0 & 0 & 0 \\
\hline Altersexuel & 0 & 1 & 1 & 1 & 0 & 0 & 0 \\
\hline Asexuel & 1 & 0 & 1 & 0 & 1 & 1 & 0 \\
\hline Bisexuel & 1 & 1 & 1 & 1 & 1 & 1 & 1 \\
\hline Bispirituel & 0 & 0 & 0 & 0 & 0 & 1 & 0 \\
\hline Cisgenre & 0 & 1 & 1 & 0 & 1 & 1 & 1 \\
\hline Gay/Gai & 1 & 1 & 1 & 1 & 0 & 1 & 1 \\
\hline Homosexuel & 1 & 1 & 1 & 1 & 1 & 1 & 1 \\
\hline Intersexe & 1 & 0 & 0 & 1 & 1 & 1 & 0 \\
\hline Intersexué & 1 & 1 & 0 & 0 & 0 & 0 & 1 \\
\hline Lesbienne & 1 & 1 & 1 & 1 & 1 & 0 & 1 \\
\hline LGBT+ & 1 & 1 & 1 & 1 & 0 & 1 & 1 \\
\hline Non-binaire & 0 & 0 & 1 & 1 & 1 & 0 & 0 \\
\hline Pansexuel & 0 & 0 & 1 & 0 & 1 & 0 & 0 \\
\hline Queer & 1 & 1 & 1 & 1 & 1 & 1 & 1 \\
\hline Trans & 1 & 0 & 1 & 0 & 1 & 1 & 1 \\
\hline Transgenre & 1 & 1 & 1 & 1 & 1 & 1 & 1 \\
\hline Transsexuel & 1 & 1 & 1 & 1 & 1 & 1 & 1 \\
\hline TOTAL & $\mathbf{1 2}$ & $\mathbf{1 2}$ & $\mathbf{1 5}$ & $\mathbf{1 2}$ & $\mathbf{1 2}$ & $\mathbf{1 2}$ & $\mathbf{1 1}$ \\
\hline
\end{tabular}

Tableau 1. Présence des appellations d'identités de genre non traditionnelles dans la nomenclature des dictionnaires à l'étude

On constate d'abord que l'étendue des nomenclatures des dictionnaires professionnels et celle des dictionnaires profanes sont comparables. Le GDT en compte le plus : un travail terminologique a été fait ces dernières années sur ce champ lexical, ce qui nous a été confirmé par une de ses terminologues. Puisqu'ils se concentrent sur ce vocabulaire, les dictionnaires profanes pourraient comporter plus de mots spécialisés, donc moins fréquents dans la presse générale et absents des dictionnaires généraux. Dans notre corpus, seul le Wiktionnaire offre plusieurs entrées de ce type : ambisexuel $\left(12^{11}\right)$, genderqueer (44, aussi dans Rainbow), neutrois (4), unisexuel (28), transpédégouine (27).

11. Les chiffres entre parenthèses après une appellation renvoient à la fréquence dans Eureka en date d'octobre 2018. 
Les deux premières appellations du tableau présentent un cas particulier : en plus du Wiktionnaire, elles sont présentes à la nomenclature de deux dictionnaires professionnels québécois, Usito et le GDT, alors que ce sont des appellations relativement rares par rapport aux plus fréquentes que nous verrons dans la section suivante (allosexuel, 366 et altersexuel, 143). Ceci s'explique par l'ancrage dans la communauté de référence : ces appellations sont proposées par l'OQLF en remplacement de l'anglicisme queer. Or l'utilisation de ces appellations dans la presse n'est pas naturelle mais souvent autonyme, ou accompagnée d'un commentaire métalinguistique : "Chez nous, le mot « allosexuel « est accepté par l'Office de la langue française en guise de traduction pour queer. » (Chartier, 2017 : A5; voir également Laprade 2014 : 4). Bispirituel, présent uniquement dans le glossaire de la CCGQ, est aussi à mettre en lien avec le contexte référentiel nord-américain, puisqu'il s'agit d'une identité de genre non binaire présente dans la philosophie des peuples autochtones ${ }^{12}$.

Nous avons recentré le reste de l'analyse sur les appellations néologiques apparues depuis l'an $2000^{13}$ dont la fréquence est la plus élevée dans Eureka, soit $L G B T$ et ses variantes, queer et trans :

\begin{tabular}{|l|c|c|c|c|c|c|c|}
\hline & PRE & Usito & GDT & Wiktionnaire & FNEEC & CCGQ & Rainbow \\
\hline LGBT+ & 1 & 1 & 1 & 1 & 0 & 1 & 1 \\
\cline { 2 - 7 } Queer & 1 & 1 & 0 & 1 & 1 & 1 & 1 \\
\cline { 2 - 5 } Trans & 1 & 0 & 1 & 0 & 1 & 1 & 1 \\
\hline
\end{tabular}

Tableau 2. Appellations d'identités de genre non traditionnelles apparues depuis 2000 et de fréquence élevée

Ces mots sont de loin les plus fréquents de notre corpus, avec des dizaines de milliers d'occurrences, et la nomenclature d'un dictionnaire spécialisé sur ce vocabulaire ne pourrait pas justifier leur absence. Or seul queer est présent dans tous les dictionnaires profanes de notre étude. Le PRÉ, le CCGQ et le Rainbow Project décrivent les trois termes. Le Wiktionnaire ne décrit pas trans, malgré l'étendue de sa nomenclature, et, fait plutôt surprenant, le glossaire de la FNEEC ne décrit pas $L G B T$, même si une variante figure sur la page de couverture du lexique et, pourrait-on déduire, dans son titre complet :

12. À la suite de notre présentation au colloque auquel ces actes sont dédiés, et après des échanges avec les représentants du Wiktionnaire qui étaient sur place, bispirituel et sa famille morpholexicale ont fait leur entrée dans le Wiktionnaire, mais pas dans le Vocabulaire LGBTIQ en français, qui fait l'objet de notre étude.

13. À titre d'exemple, la première occurrence de LGBT que nous ayons trouvée dans les journaux francophones date de 2002: « Il doit y avoir un débat politique et une réflexion sur 1'homoparentalité », a estimé pour sa part René Lalement, porte-parole de l'association Inter-LGBT (AFP Infos Françaises Général, 4 mars 2002). 


\section{LGBTQI2SNBA+ \\ Les mots de la diversité liée au sexe, au genre et à l'orientation sexuelle}

Une autre absence surprenante est celle de queer dans le GDT, qui a pourtant fait un travail récent sur ce vocabulaire. Queer apparaît uniquement en tant que terme anglais dans le GDT, et lorsqu'on tape queer dans la barre de recherche, on arrive automatiquement sur la fiche de allosexuel, appellation très peu fréquente dans la presse générale et contestée par certains groupes de défense de droits des personnes LGBT, comme ici, la directrice générale du Conseil québécois LGBT : "Marie-Pier Boisvert considère que altersexuel et allosexuel demeurent des équivalents français de queer peu convaincants et très peu utilisés. » (Vigneault, 2016 : pause 4) Ici, les préoccupations normatives prennent le pas sur les préoccupations identitaires et sur la fréquence d'utilisation du mot. Les équivalents proposés ne sont pas repris par la population. Par ailleurs, queer comble une lacune lexicale : son signifié est délibérément flou, afin d'être le plus inclusif possible.

\footnotetext{
"Queer" est [...] une manière de chercher à dissoudre les frontières afin que d'autres identités (transgenres, bisexuels, etc.) et la multiplicité des identités gays et lesbiennes (folles, tantes, butch/fem...) aient toute leur place dans un mouvement contestant les normes sexuelles, culturelles et sociales (Éribon, 2003 : 394)
}

Enfin, le Rainbow Project a choisi la forme Trans personne(s) pour l'entrée trans, ce qui pose problème car il ne s'agit pas d'une forme en usage - aucune occurrence n'a été relevée dans Eureka.

\section{2. Le traitement définitoire des appellations les plus fréquentes}

Pour ce qui est du traitement définitoire, nous avons examiné les sèmes fournis par les dictionnaires. Respectent-ils les éléments de sens importants relevés par les groupes de défense des droits des personnes LGBT? Nous confronterons les définitions avec les informations fournies par ces groupes, tel que relevé dans la presse par exemple.

\section{2. 1. LGBT}

L'appellation la plus fréquente dans la presse générale est $L G B T$. Elle est utilisée par plusieurs organisations (par exemple, elle est choisie pour le nom du Conseil québécois LGBT). Ce qui semble militer en sa faveur est son caractère inclusif. En tant qu'appellation parapluie, $L G B T$ (et ses variantes) englobe à la fois des notions d'identité de genre et d'orientation sexuelle. L'enjeu principal avec la définition de ce mot est de conserver ce caractère inclusif, perceptible dans la forme même de l'appellation, qui permet d'ajouter autant de lettres (et de chiffres) que nécessaire. 
$L G B T$ (ou une variante) est présent dans les six dictionnaires présentés dans le tableau 3. Notons qu'il y a lieu de se questionner sur le choix de l'entrée LGB dans le Rainbow Project, variante beaucoup moins fréquente que LGBT, qui est d'ailleurs employée dans le lexique :

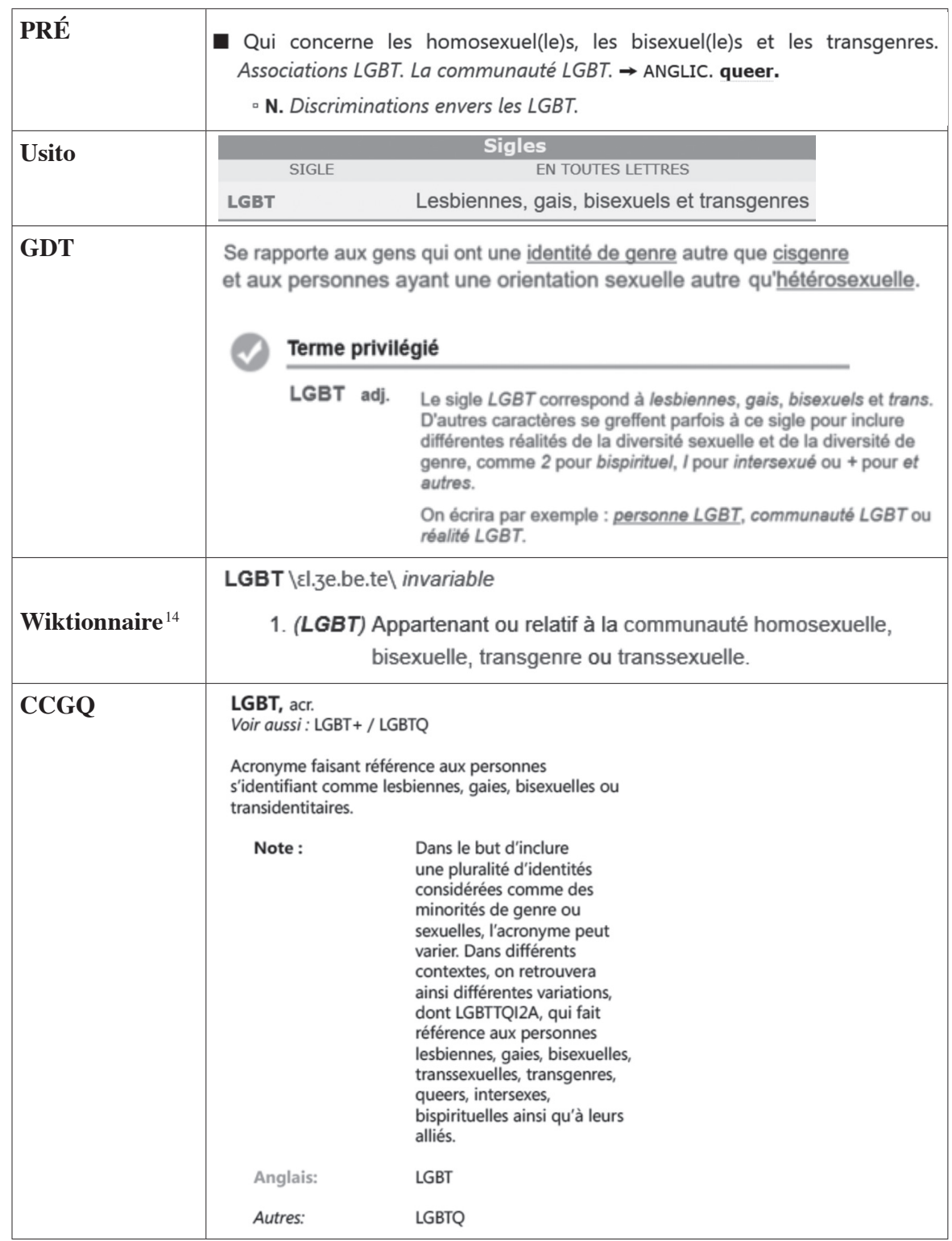

14. Le Wiktionnaire a des entrées différentes pour LGBTI, LGBTIQ, etc.; chaque fois, le traitement est augmenté par l'explicitation de la lettre. Par exemple, pour l'entrée LGBTI : (LGBT) «Appartenant ou relatif à la communauté homosexuelle, bisexuelle, transgenre ou intersexe. » 


\begin{tabular}{|l|l|}
\hline Rainbow & $\begin{array}{l}\text { LGB (adj.) Abréviation, communément utilisée, pour « Lesbienne (L), Gay } \\
\text { (G) ou Bisexuel (B) ». Souvent un T signifiant Trans y est ajouté, ce qui } \\
\text { donne LGBT. On peut également le retrouver suivi de l'acronyme QQIA qui } \\
\text { signifie "Queer, Questioning, Intersex and Allies " (en français " Homo, } \\
\text { Questionnement, Intersexué et Alliés ") }\end{array}$ \\
\hline
\end{tabular}

Tableau 3. Traitement définitoire de $L G B T$

Un problème survient si la définition est trop restrictive, ou si elle se cantonne à l'énumération des mots identifiés par une lettre dans le sigle, surtout si on ne mentionne pas que le nom peut se voir greffer d'autres lettres. Or c'est le cas des définitions du PRÉ, du Wiktionnaire et de la CCGQ. En cela, elles ne diffèrent guère du traitement d'Usito, où LGBT figure en tant que sigle, dans un tableau, sans recevoir de définition. Cependant, dans une note, le glossaire de la CCGQ précise que «l'acronyme peut varier ». La définition du Rainbow Project est également construite à partir du sigle, et si on y précise que des lettres peuvent être ajoutées, on ne comprend pas pourquoi ce sont des exemples d'appellations anglaises qui sont données («Queer, Questioning, Intersex and Allies »). Le seul ouvrage qui offre un traitement définitoire satisfaisant est le GDT : plutôt que de se limiter à énumérer les appellations identifiées par les lettres, il donne une définition complète qui respecte la vocation parapluie de l'appellation. C'est dans la note que des explications sur la formation du sigle sont données.

\subsubsection{Trans}

L'appellation trans désigne une identité de genre. À l'origine, trans est une apocope : il vient de transsexuel et de transgenre. Marie-Pier Boisvert, citée plus haut, considère ce terme comme étant inclusif et suggère d'utiliser trans plutôt que de faire la distinction entre transgenre et transsexuel :

« Il [trans] intègre toutes les personnes non binaires, c'est-à-dire celles qui ne s'affichent ni comme homme ni comme femme. Ou les deux. Ou entre les deux. Alors quand on parle d'une personne trans, c'est un terme parapluie qui peut désigner toutes ces personnes. » (Marie-Pier Boisvert citée dans Vigneault, 2016 : pause 4)

Le traitement définitoire doit donc conserver ce caractère inclusif. Trans est absent d'Usito et du Wiktionnaire, alors que c'est la $2^{\mathrm{e}}$ appellation d'identité de genre non traditionnelle la plus fréquente dans la presse générale. 


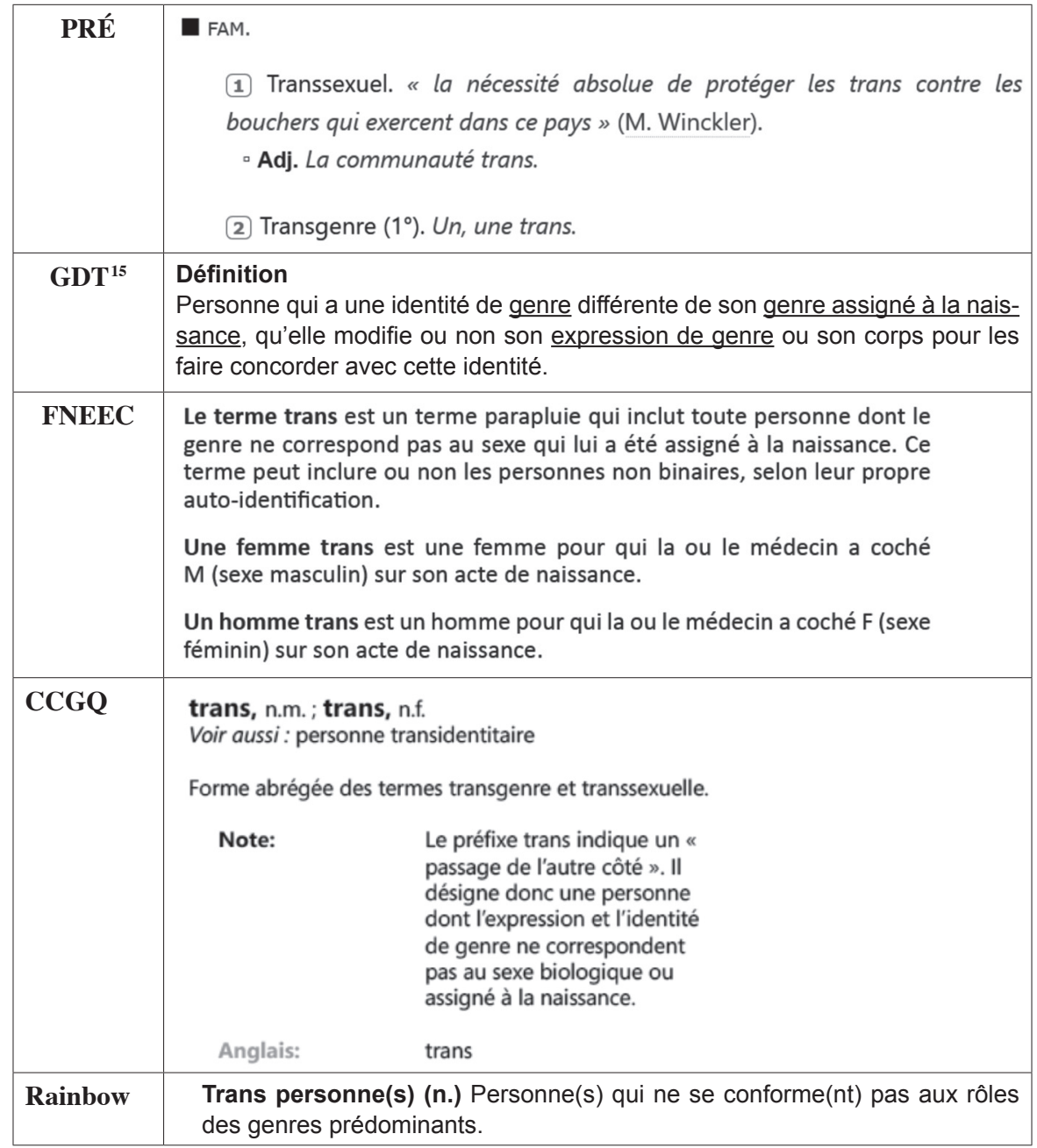

Tableau 4. Traitement définitoire de trans

Les définitions du GDT et des lexiques de la FNEEC et du Rainbow Project respectent l'inclusion des sens de transgenre et de transsexuel. Cependant, dans le glossaire de la CCGQ, c'est dans la note et non dans la définition que se trouve l'information. On ne peut pas considérer « Forme abrégée des termes transgenre et transsexuelle » comme une définition en tant que telle; elle ne fournit aucun sème, et sert à expliquer la formation du mot plutôt que son signifié. Pour trans, c'est encore le GDT qui offre le traitement définitoire le plus satisfaisant.

15. L'entrée dans le GDT est « personne trans », mais on peut accéder à la fiche en tapant uniquement trans. 


\section{2.3. Queer}

En anglais, queer signifie à l'origine "étrange » et a d'abord été utilisé en tant qu'insulte homophobe. «Le mouvement gai s'est ensuite réapproprié le terme dans un geste de retournement du stigmate. » (Laprade, $2014: 3$ ). Le mot est aujourd'hui employé de manière parfois large pour désigner tout ce (et toutes celles et ceux) qui s'écarte de l'hétéronormativité et de la binarité des genres :

Avec le temps, les travaux et les auteurs se sont multipliés, et le vocable queer a fini par recouvrir une grande variété de positions théoriques dont les contours sont difficiles à établir : ils réfèrent à l'idée d'une fluidité, d'une suspension de l'identité; à la remise en question de la viabilité et de l'utilité politique des catégories de l'identité sexuelle; à la transgression des normes sexuelles, à la multiplication des identités et des pratiques marginales. Être queer, c'est brouiller les frontières, mélanger les genres, promouvoir l'instabilité et l'indécidabilité des identités. (St-Hilaire, 1999 : 27)

Les définitions de queer doivent donc conserver cette ouverture du mot, ce flou. Queer est traité par tous les dictionnaires à l'étude sauf le GDT :

\begin{tabular}{|c|c|}
\hline PRÉ & $\begin{array}{l}\text { ANGLIC. Personne dont l'orientation ou l'identité sexuelle } \\
\text { ne correspond pas aux modèles dominants. } \rightarrow \text { bisexuel, } \\
\text { homosexuel, transgenre, transsexuel. Un, une queer. } \\
\text { Les queers. } \\
\text { - Adj. Activistes queers. La communauté queer. } \rightarrow \text { LGBT. }\end{array}$ \\
\hline Usito & $\begin{array}{l}\text { queer [kwi:R] n. et adj. } \\
\text { Personne qui ne se définit pas par les catégories traditionnelles de } \\
\text { genre et d'orientation sexuelle. } \\
\Rightarrow \text { AlLosexuel, ALTERSEXUEL. } \\
\text { Un queer, une queer. } \\
\text { "les queers sont des personnes qui ne veulent pas être } \\
\text { étiquetées comme étant un homme, une femme, un } \\
\text { hétérosexuel ou un homosexuel. Pour ces gens, il y a une } \\
\text { fluidité dans les identités sexuelles et de genre. Ils ne } \\
\text { veulent pas qu'on les catalogue » (La Presse plus, 2016). } \\
\text { - Adj. Personne queer. Mouvement queer. } \\
\text { REM. L'emploi de queer est parfois critiqué comme synonyme non standard de } \\
\text { allosexuel, altersexuel. - À l'origine, en anglais, ce mot était utilisé de manière } \\
\text { péjorative. } \\
\text { ÉTYMOLOGIE } \\
\text { L'étymologie de cet article est en cours de révision. }\end{array}$ \\
\hline
\end{tabular}




\begin{tabular}{|c|c|c|c|c|}
\hline \multirow[t]{7}{*}{ Wiktionnaire } & \multicolumn{4}{|c|}{ Adjectif [modifier le wikicode ] } \\
\hline & \multicolumn{2}{|c|}{ queer $\backslash \mathrm{kwis} \backslash$ invariable } & Singulier & Pluriel \\
\hline & \multirow{2}{*}{\multicolumn{2}{|c|}{$\begin{array}{l}\text { 1. (Anglicisme) Qualifie ce qui n'est pas hétéronormé. Notamment : Gay, lesbienne, } \\
\text { transgenre, allosexuel, altersexuel, pansexuel... } \\
\text { • Le mouvement queer. }\end{array}$}} & queer & queers \\
\hline & & & \multicolumn{2}{|c|}{$\mid$ kwi६ $\backslash$} \\
\hline & \multirow{3}{*}{\multicolumn{4}{|c|}{ 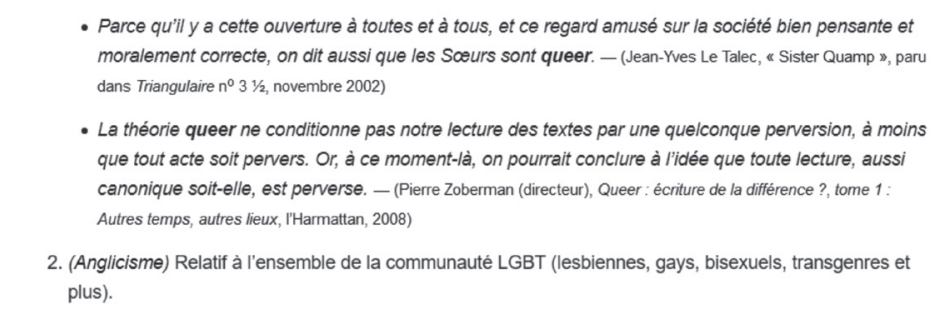 }} \\
\hline & & & & \\
\hline & & & & \\
\hline FNEEQ & \multicolumn{4}{|c|}{$\begin{array}{l}\text { Une personne queer est une personne qui choisit ce terme pour affirmer } \\
\text { son orientation sexuelle, son identité de genre ou son expression de } \\
\text { genre. La réappropriation de ce terme, autrefois considéré comme } \\
\text { péjoratif, est une forme d'empowerment. Certaines personnes queers } \\
\text { incluent un aspect politique de rejet des normes sociales dans cette auto- } \\
\text { identification. }\end{array}$} \\
\hline \multirow[t]{4}{*}{ CCGQ } & \multirow{2}{*}{\multicolumn{4}{|c|}{$\begin{array}{l}\text { queer, n.m.; queer, n.f. } \\
\text { Voir aussi : altersexuel, allosexuel } \\
\text { Personne qui n'adhère pas à la division binaire } \\
\text { traditionnelle des genres et des sexualités, s'identifiant } \\
\text { à une identité de genre ou à une orientation sexuelle } \\
\text { non-conforme ou fluide. }\end{array}$}} \\
\hline & & & & \\
\hline & Note: & \multicolumn{3}{|l|}{$\begin{array}{l}\text { Terme d'origine anglo- } \\
\text { saxonne, réapproprié par } \\
\text { les communautés LGBT de } \\
\text { manière à en faire un symbole } \\
\text { d'auto-détermination et } \\
\text { de libération plutôt qu'une } \\
\text { insulte, qui fait référence } \\
\text { à toute personne dont } \\
\text { l'identité ou les pratiques } \\
\text { vont à l'encontre des normes } \\
\text { structurant le modèle social } \\
\text { hétéronormatif. En ce sens, le } \\
\text { terme connote d'une auto- } \\
\text { représentation contestataire. }\end{array}$} \\
\hline & Anglais: & \multicolumn{3}{|l|}{ queer } \\
\hline Rainbow & \multicolumn{4}{|c|}{$\begin{array}{l}\text { Queer ("homo") (n.) Autre terme désignant les LGBT, il est également uti- } \\
\text { lisé de manière péjorative; c'est pourquoi tous les gays ou lesbiennes s'en } \\
\text { détachent, bien que beaucoup de jeunes LGBT l'utilisent comme une affir- } \\
\text { mation. }\end{array}$} \\
\hline
\end{tabular}

Tableau 5. Traitement définitoire de queer

Les définitions du PRÉ, d'Usito, du Wiktionnaire et du lexique de la CCGQ sont suffisamment larges pour respecter l'essence de l'appellation queer : on 
évoque partout l'orientation sexuelle et l'identité de genre, et on emploie une définition par exclusion (qui n'adhère pas, qui ne se définit pas...).

Usito fait des renvois à des synonymes très peu attestés dans la presse et qui ne sont pas utilisés par les groupes concernés (allosexuel, altersexuel). La motivation derrière ces renvois s'explique probablement par les préoccupations normatives du dictionnaire, qui centre sa description sur le français québécois standard, et qui porte une attention particulière aux anglicismes (voir Cajolet-Laganière, 2017). On peut tout de même se questionner sur le fait de renvoyer à des recommandations qui, même si elles proviennent d'un organe linguistique officiel, n'ont pas réellement pris racine dans l'usage, notamment chez les groupes concernés.

La définition proposée par la FNEEC est trop large : on pourrait l'utiliser pour définir hétérosexuel, lesbienne, homosexuel, LGBT, bref, toutes les appellations d'identités de genre ou d'orientation sexuelle traditionnelles ou non. Enfin, le traitement définitoire du Rainbow Project ne tient pas compte du fait que certaines personnes et groupes revendiquent cette désignation - la dernière partie de la définition («bien que beaucoup de jeunes LGBT l'utilisent comme une affirmation ») étant en opposition totale avec ce qui précède. Les autres dictionnaires qui mentionnent l'origine péjorative du mot la mettent en note, en remarque, ou, comme le PRÉ, dans la rubrique étymologique.

\section{CONCLUSIONS}

Si les lexiques profanes comptent plus d'appellations d'identité de genre non traditionnelle à leur nomenclature, pour les termes les plus fréquents, les nomenclatures sont comparables, des dictionnaires professionnels s'en tirant honorablement, et des dictionnaires profanes présentant certaines lacunes. Par contre, lorsqu'on examine les entrées, les dictionnaires profanes présentent plusieurs problèmes. Si la pénétration dans le dictionnaire vaut comme signe de reconnaissance, que penser lorsqu'une appellation, même fréquente, est absente? Cette question se pose particulièrement pour les deux lexiques à vocation pédagogique (Rainbow Project et FNEEQ). Le traitement définitoire est également comparable, avec des lacunes et des descriptions satisfaisantes dans les deux types d'ouvrages.

Les dictionnaires profanes sont-ils tenus à la même rigueur que les dictionnaires professionnels? Nous pensons que oui ${ }^{16}$, puisqu'ils sont les seuls dépositaires de certaines appellations moins fréquentes, et qu'ils ont pour but de donner une légitimité aux groupes dominés. L'usager ne fait probablement pas de distinction selon l'équipe derrière le projet, professionnelle ou non.

Admettant que le temps entre la création d'un néologisme et sa pénétration dans le dictionnaire raccourcit (Pruvost et Sablayrolles, 2003), surtout lorsqu'on a des

16. Voir les actes de la première édition de ce colloque pour plusieurs points de vue sur cette question : http://www.dorif.it/ezine/show_issue.php?iss_id=25 
dictionnaires professionnels en ligne (Usito, GDT), et que parmi les dictionnaires étudiés, c'est un dictionnaire professionnel, le GDT, qui présente la nomenclature et le traitement lexicographique les plus complets, on peut se demander quelle est la plus-value des dictionnaires profanes. La réponse se trouve peut-être du côté d'une collaboration entre lexicographes profanes et professionnels.

Les dictionnaires professionnels ont déjà des protocoles de travail leur permettant de faire des recherches pour chaque champ lexical. Le fait qu'ils soient maintenant disponibles en ligne facilite les changements, à tous les niveaux du traitement (nomenclature, définition, exemples, marques...) et accélère le processus de révision. Si un groupe ressent le besoin de créer un lexique ou un glossaire sur une thématique particulière, pourquoi ne pas regarder ce que les dictionnaires professionnels ont déjà fait à ce sujet? Les définitions doivent-elles nécessairement être originales, ou ne peut-on pas envisager de reprendre, en les citant, les définitions convenables des ouvrages professionnels? Si on constatait un manque, les lexicographes profanes pourraient aider à le combler, que ce soit par des mécanismes de lexicographie collaborative ou par des contacts plus balisés entre les lexicographes professionnels et les usagers ${ }^{17}$. La collaboration pourrait aller dans les deux sens, comme le soulignent ici Sajous et Hathout :

De plus, si l'informatique facilite la détection de créations lexicales par les lexicographes, la néologie sémantique et les changements de connotation sont plus complexes à automatiser. L'aide des internautes peut dans ce cas se révéler utile en amont de l'analyse du lexicographe. Enfin la diversité des domaines plus ou moins spécialisés pénétrant la vie quotidienne, dont le vocabulaire a vocation à intégrer la nomenclature des dictionnaires, rend utile le recours à la pluralité des expertises des internautes, dont les connaissances complètent la compétence lexicographique des professionnels des dictionnaires. (Sajous et Hathout, 2017 : 29)

Tout comme les outils informatiques et électroniques avant elle, la lexicographie profane en ligne bouscule les façons de faire traditionnelles en lexicographie. Cette dernière devrait peut-être emboîter le pas pour enrichir sa propre pratique, à l'intérieur des balises de la métalexicographie, qui permettent des dictionnaires de qualité.

Mireille ELCHACAR Université Téluq

\section{RÉFÉRENCES BIBLIOGRAPHIQUES}

BOURDIEU, P. 2001. Langage et pouvoir symbolique, Paris, Éditions Fayard.

CAJOLET-LAGANIĖRE, H. 2017. " Le traitement des anglicismes critiqués dans le dictionnaire en ligne Usito », Les anglicismes : des emprunts à intérêt variable?, Québec, Office québécois de la langue française, 128-150.

17. Le lexique de la CCGQ évoque une collaboration avec l'OQLF : « Ce projet a été possible grâce à l'appui de l'Office québécois de la langue française (OQLF) », et précise que le lexique sera disponible via le site de l'OQLF : http://gdt.oqlf.gouv.qc.ca. Il aurait été intéressant d'avoir plus de détails sur ce partenariat. 
CHARTIER, S. 2017. " "Queer", un flou clair pour les minorités sexuelles », Le Devoir, 20 juillet, A5.

ELCHACAR, M. et SALITA, A. 2018. «Les appellations des identités de genre non traditionnelles. Une approche lexicologique », Langage et société, $\mathrm{n}^{\circ} 165$, 139-165.

ÉRIBON, D. 2003. Dictionnaire des cultures gays et lesbiennes, Paris, Larousse.

GRECO, L. 2015. "Présentation : la fabrique des genres et des sexualités », Langage et société, $\mathrm{n}^{\circ} 152,7-16$.

LAPRADE, B. 2014. "Queer in Québec : étude de la réception du mouvement queer dans les journaux québécois », Cygne noir 2, [en ligne], http://www. revuecygnenoir.org/numero/article/queer-in-quebec\#body, consulté le 3 mai 2017.

PETIT, G. 2012. «Présentation : la dénomination » Langue française, ${ }^{\circ}$ 174, 3-9.

PRUVOST, J. et SABLAYROLLES, J.-F. 2003. Les Néologismes, Collection «Que Sais-je? », 3674, Paris : Presses universitaires de France.

SAJOUS, F. et HATHOUT, N. 2017. «Informativité, neutralité et point de vue dans une offre dictionnairique hétérogène : vers une complémentarité ? », Revue française de linguistique appliquée, Vol. XXII, 27-40.

ST-HILAIRE, C. 1999. " Les paradoxes de l'identité et le devenie-queer du sujet : de nouveaux enjeux pour la sociologie des rapports sociaux de sexe », Recherches sociologiques, Université Catholique de Louvain, vol. XXX, $\mathrm{n}^{\circ} 3$, 23-42.

VIGNEAULT, A. 2016. « Lexique LGBTQ », La Presse, 9 août, pause 4.

VINCENT, N. 2017. Présence et description d'emplois québécois dans des dictionnaires disponibles gratuitement en ligne, «Repères DoRiF n. 14 - Dictionnaires, culture numérique et décentralisation de la norme dans l'espace francophone », DoRiF Università, Roma, http://www.dorif.it/ezine/ezine_articles.php?id=379 